\title{
Disseminated Acquired Reactive Perforating Collagenosis Associated with Longlasting HBV infection, Cirrhosis and Sigmoid Colon Adenoma \\ B Tas ${ }^{1}, \mathrm{~K}_{\text {Turker }}{ }^{2}, \mathrm{E} \mathrm{Tas}^{3}, \mathrm{AH} \mathrm{Ozakyol}^{4}, \mathrm{~A} \mathrm{Caglar}^{5}$
}

\begin{abstract}
One of the perforating dermatoses (PD) is acquired reactive perforating collagenosis (ARPC). Clinical presentations of ARCP are umbilicated papules or nodules with a central adherent keratotic plug containing cellular debris and collagen. The disease group is histopathologically characterized by transepidermal elimination of various dermal substances into cup-shaped epidermal invagination. PD is usually associated with diabetes mellitus (DM) or chronic renal failure (CRF). In recent years, it has also been reported to be associated with some other metabolic, endocrinological and infectious diseases and malignancies. Herein, we report a 62-year- old woman with ARPC whose lesions were associated with longlasting chronic hepatitis B virus (HBV) infection, cirrhosis, and sigmoid colon displastic adenoma. To the best of our knowledge these associations have not been reported previously.
\end{abstract}

Keywords: Adenoma, cirrhosis, hepatitis B virus, sigmoid colon, skin diseases

From: ${ }^{1}$ Department of Dermatology, and ${ }^{2}$ Department of Infectious Diseases, Bagcilar Research and Training Hospital Istanbul/Turkey, ${ }^{3}$ Department of Gastroenterology, Dumlupınar University, Kütahya Evliya Celebi Research and Training Hospital, Kütahya/Turkey, ${ }^{4}$ Department of Gastroenterology, Eskişehir Osmangazi University, Eskişehir/Turkey, ${ }^{5}$ Department of Pathology,

Bagcilar Research and Training Hospital Istanbul/Turkey

Correspondence: Dr B Tas, Director of Dermatopathology Department, Address: Atakoy 7-8. K1sım, Martı Sitesi, 14/105, postal code: 34156, Bakırkoy/Istanbul, Fax: (90) 212-4404000, e-mail: betulavc@yahoo.com 


\section{INTRODUCTION}

Reactive perforating collagenosis (RPC) is a rare cutaneous disorder of unknown etiology (1). The prevalence, incidence and the exact pathogenesis of this rare dermatosis are unknown (1-3). The sex distribution is usually equal. Its peak generally occurs in the 6th decade of life. The pathogenesis of the disease was originally described by Mehregan et al. in 1967. They proposed that when susceptible patients are exposed to mild superficial trauma, the dernal collagen go into necrobiosis, which then discarded through the process of transepidermal elimination. There are two forms of the disease. While the childhood form is inherited as autosomal recessive, the adult form is associated with some acquired diseases (3).

\section{CASE REPORT}

A 62-year-old Caucasian woman was admitted to our dermatology clinic due to her severe itching, and disseminated skin lesions. According to the patient's medical history, the patient had undergone HBV infection for 12 years ago, but she did not develop anti hepatitis B antibodies. However, her HBV DNA level was not high enough to receive any antiviral treatments. Two years ago, the patient developed anti HBV antibodies. However, 1 year ago, hepatic condition of the patient worsened. She was admitted to a gastroenterologist 8 months ago due to the severe itching and abdominal swelling and was diagnosed with chronic hepatic failure and cirrhosis. The patient had no additional known disease and she was not under any treatment excluding antihistamines. She also stated that she has had some digestive problems and constipation for three months. Her skin lesions first occured 6 months ago, and were initially localized on the elbows and knees. In six months, they gradually progressed to her present state. In the dermatological examination, the skin was dirty yellow in color, and 
multiple scratch marks as well as many round, purple umblicated papulonodular lesions were seen. Most of the lesions complied with the scratch marks. These Kobneriform lesions were especially located on the back, and extensor surfaces of the rest of the body and extremities, sparing the face, palms, soles and anterior surface of the trunk. The lesions were in the shapes of the cup, and $3-6 \mathrm{~mm}$ in diameter. In the center of the lesions, there were whitish-grey and cretaceous plugs. Additionally, there were prominent abdominal vessels and a lot of striae on the abdominal wall (Figure 1a,b,c,d). From one of the most prominent lesions, an excisional skin biopsy was taken. Histopathological examination of the biopsy specimen showed, a vertically-settled invaginated area which was composed of an epidermal hyperkeratosis and acanthosis. In the center of the cup-shaped lesion, prominent basophilic degeneration, necrosis of dermal collagen, and mixed inflammatory infiltrate including a lot of macrophages in the mid-dermis were seen (Figure 2a,b). Histochemical examination (with Masson trichrome) showed coarse and degraded eosinophilic collagen bundles beneath the ulcer base and in the mid dermis (Figure 2c,d). In the physical examination, an ageappropriate hypertension, prominent hepatomegaly, abdominal distantion, marked abdominal vessels, lower extremity edema and moderate heart failure were detected. There was no regional lymphadenomegaly. The laboratory examinations indicated the followings: blood glucose; $96 \mathrm{mg} / \mathrm{dl}(\mathrm{N}: 82-115)$, urea; $48 \mathrm{mg} / \mathrm{dl}$ (N:17-49), creatinin; $0.8 \mathrm{mg} / \mathrm{dl}$ (N:0.5-0.9), calcium;7.9 mg/dl (N:8.7-10.4), potassium; $6.49 \mathrm{mmol} / \mathrm{L}(\mathrm{N}: 3.5-5.1)$, albumin; $3.3 \mathrm{~g} / \mathrm{dl}$ (N:3.5-5.2), C-reactive protein; $16.93 \mathrm{mg} / \mathrm{L}(\mathrm{N}: 0-5)$, protrombin time; $18.7 \mathrm{sn}(\mathrm{N}: 11-16)$, protrombin activity; $42.2 \%$ (N:70-130), international normalized ratio; 1.63 (0.8-1.3), blood pO2; 30.7 mmHg (N:71-104), O2Hb; 42.4 (N:95-99), hematocrit; 25RU (N:34.8-50), gamma glutamyl transferase; $105 \mathrm{U} / \mathrm{L}(\mathrm{N}: 6-42)$, anti-HBs; $62.521 \mathrm{mlU} / \mathrm{ml}(\mathrm{N}:<10$ negative), CA125; 496.1 U/ml (N:<35), lactate dehydrogenase; $311 \mathrm{U} / \mathrm{L}(\mathrm{N}: 135-214)$, amylase; $131 \mathrm{U} / \mathrm{L}(\mathrm{N}: 28$ 100), and D-dimer; $1.23 \mathrm{ug} / \mathrm{L}(\mathrm{N}: 0-0.5)$. The stool examination revealed an occult bleeding. 
In the abdominal ultrasonograpy (USG), hepatomegaly, heterogeneity and diffuse granular structures in the liver parenchyma, increased echogenicity in the periportal areas, and massive ascites surrounding the perisplenic and paracolic regions, and in the douglas pouch were detected. Doppler USG of the portal vein was normal. She underwent a computed tomography scan showing similar findings to abdominal USG in addition to mesenteric heterogeneity and degenerative changes in bone structures. In most relevant consultations, no accompanying clinical pathologies such as diabetes mellitus, kidney, and genitourinary system diseases were detected. In the requested gastroenterology consultation, to determine the nature of abdominal fluid collection, a traditional peritoneal paracentesis was performed, and aspirated fluid pathology revealed that the patient has malignant ascites. Thereafter, a trucut liver biopsy was performed, and its pathological examination showed a massive sinusoidal dilation, perivascular fibrosis, proliferation of bile canaliculi, degeneration of hepatocytes, and focal cholangitis. Performed endoscopic biopsy showed a moderate metaplasia in the gastric antrum, and in the following colonoscopia, three, grade-2 tubular displastic sigmoid adenoma were detected. All other laboratory parameters were within normal limits. With the clinical and pathological findings, and in view of available laboratory investigations, the patient was diagnosed with cirrhosis, malignant ascites and sigmoid colon adenoma with omental metastasis, and the skin lesions were diagnosed with ARPC. The patient underwent colonoscopic polypectomy, and sequential peritoneal dialysis. Because the patient did not give consent, no additional adjuvant chemotherapy was applied The skin lesions were treated with topical and oral antihistamines and topical corticosteroids. The patient is still in the third month of follow up period, and unfortunately the hepatic and general condition has been worsening. However, the number and severity of the skin lesions have moderately decreased after removal of the tumors and 4 sequential peritoneal dialysis, but not completely. 


\section{DİSCUSSİON}

PD is a very complicated group of diseases clinically and histopathologically. They may be classified into primary and secondary types. The primary PDs (Kyrle disease, elastosis perforans serpiginosa (EPS) and reactive perforating dermatosis (or RPC) ) result from perforation of the dermoepidermal junction and transepidermal elimination of collagen or elastic fibers. The secondary forms (granuloma annulare, calcinosis cutis, necrobiosis lipoidica, chondrodermatitis nodularis chronica helicis and keratoacanthoma) are usually detected during the course of another dermatosis with different pathogenesis. The perforation, and elimination of degenerated dermal connective tissue occur less frequently (3). RPC is one of the primary PDs $(2,3)$. It is usually intensely itchy, shows Köbner's phenomena and primarly located on the extensor surface of extremities. (1). In acquired ARPC, the lesions usually arise in adults over 18 years of age (3). A typical lesion appears as a small, purple, and umblicated papul or nodule whose center is filled with a cretaceous plug. These lesions can be found in different stages of development. Histopathologically, prominent epidermal hyperkeratosis and acanthosis of the perforated and invaginated epidermis in addition to basophilic degeneration, collagen necrosis and mixed inflammatory infiltrate including macrophages in the dermis are seen (1-3).The clinical and histopathological features of our patient complied with the literature. In the etiopatogenesis, suggested causes include a genetic predisposition (3), related diseases such as DM or CRF (1-3) and occasionally drugs such as clopidogrel, indinavir and sirolimus. It has been stated that in patients with abnormal liver methabolism, ARPC lesions can be trggered because of the breakdown of these medications by hepatogenic cytochrome P450 (3). ARPC can occur in up to $11 \%$ of dialysis patients (2). However, in recent years it has been reported to be associated with some diseases and healthy conditions such as non-diabetic hemodialysis, endocrinological disorders, tuberculosis, pulmonary aspergillosis, neurodermatitis, acuta leukemia, Hodgkin's lymphoma, acquired 
immune deficiency syndrome, atopic dermatitis, hepatic disorders, scabies, herpes zoster infection, and pregnancy $(1,2)$. Others have speculated that the presence of some microangiopathies can make patients more susceptible to ARPC (2). Due to the reported association with both some myeloproliferative disorders and solid tumors, it can also be considered a facultative paraneoplastic disorder. It can be seen after curettage of seborrheic keratosis (3). Our patient did not have classical comorbid conditions such as DM, CRF or take the mentioned drugs but had longlasting chronic HBV infection, hepatic failure /cirrhosis and sigmoid colon adenomas, and these associations have not been reported previously. In the etiopathogenesis, it has been stated that the lesions may be a cutaneous response to superficial trauma caused by intense scratching. In the papillary dermis, histochemically altered but structurally normal, type IV collagen is present, which is surrounded by invaginated epidermal proliferation. Then, a central plug containing inflammatory cells, keratinous material and altered collagen develops, and the altered collagen is eliminated by transepithelial migration (1). Immunohistochemical studies have shown significance of growth factors and enzymes in the extracellular matrix, which are still being debated (3). On the other hand, transepidermal elimination may occur as a primary process in some PDs such as EPS, Kyrle's disease, and ARPC (4). A $67 \mathrm{kDa}$ elastin receptor ( $\beta$-galactosidase $[\beta$-gal] ) plays a crucial role in cell migration and tissue organization in the course of the development and metastasis of tumor cells (5). It is found in basal lamina which has similar affinity to that of elastin $(4,6)$. Recently, Fujimato et al. demonstrated that the 67$\mathrm{kDa}$ elastin receptor can be found in altered elastic fibres of the eliminated epidermis in EPS, suggesting that the elastin-keratinocyte interaction can play a role in the transepidermal elimination $(3,4,6)$. On the other hand, senescence-associated $\beta$-gal activity (SA- $\beta$-gal) is an important biomarker for cellular senescence, because it has been suggested that increase of SA- $\beta$-gal may contribute to the degraded connective tissue and decreased skin fibroblast 
proliferation. This in turn may lead to the wound not healing completely $(4,7)$. Indeed, it has been demonstrated that proliferative capacity and growth of skin fibroblasts were significantly inhibited by some products like cigarette extracts which cause significantly increased SA $\beta$-gal activity in the fibroblasts. It has also been showed that smoking inhibited superoxide dismutase and glutathione peroxidase activities, and increased the level of reactive oxygen species (ROS). ROS production rises in many pathological conditions (8). Similar to previously mentioned interactions, in our case, we thought that an increasing level of oxidative distress and subsequent increase in ROS during the chronic hepatocellular damage, and additional carcinogenic process could have been responsible for acceleration of an early tissue senescence, inhibition of proliferation and sequential morphological changes of skin fibroblasts, and degrading connective tissue with the increase of SA $\beta$-gal activity. Thus, we think that because of the relatively damaged immune system and decreased numbers of apoptosis, these damaged cells could be accumulated in the most stressful dermal foci, and could not be completely eliminated from the dermis. As a result of the process, the lesions of our patient may have been developed with the contribution of a Köbner reaction induced by scratching. However, we do not know whether there was any increased SA $\beta$-gal activity or additional factors that could contribute to this dissemination. Due to the moderate regression of the lesions after the removal of the tumors and peritoneal dialyses, we propose that the accompanying carcinogenesis and hepatic pathologies may be responsible for both the formation and dissemination of the lesions. However, further investigation will be needed to clarify this issue. In the differential dignosis, the other primary and secondary PDs should be considered. To satisfying therapeutic response, the management of associated disease is crucial (2). To the best of our knowledge, our patient is the first ARPC case in literature regarding these etiopathological relevances. In conclusion, 
we suggest that especially in cases of disseminated ARPC lesions, chronical or longlasting liver disorders or malignancy must be kept in mind.

Acknowledgments: None

Conflict of interests: None 


\section{REFERENCES}

1. Yadav MK, Sangal BC, Bhargav P, et al. Reactive perforating collagenosis. Indian J Pathol Microbiol 2009;52:106-107.

2. Kreuter A, Gambichler T. Acquired reactive perforating collagenosis. CMAJ 2010;182:E184.

3. Wagner G, Sachse MM. Acquired reactive perforating dermatosis. J Dtsch Dermatol Ges 2013;11:723-730.

4. Fujimoto N, Tajima S, Ishibashi A. Elastin peptides induce migration and terminal differentiation of cultured keratinocytes via $67 \mathrm{kDa}$ elastin receptor in vitro: $67 \mathrm{kDa}$ elastin receptor is expressed in the keratinocytes eliminating elastic materials in elastosis perforans serpiginosa. J Invest Dermatol 2000;115:633-639.

5. Hinek A. Biological roles of the non-integrin elastin/laminin receptor. Biol Chem $1996 ; 377: 471-480$.

6. Fujimoto N, Akagi A, Tajima S, et al. Expression of the 67-kDa elastin receptor in perforating skin disorders. Br J Dermatol 2002;146:74-79.

7. Johnen C, Hartmann B, Steffen I, et al. Skin cell isolation and expansion for cell transplantation is limited in patients using tobacco, alcohol, or are exhibiting diabetes mellitus. Burns 2006;32:194-200.

8. Yang GY, Zhang CL, Liu XC, et al. Effects of cigarette smoke extracts on the growth and senescence of skin fibroblasts in vitro. Int J Biol Sci. 2013;9:613-623. 

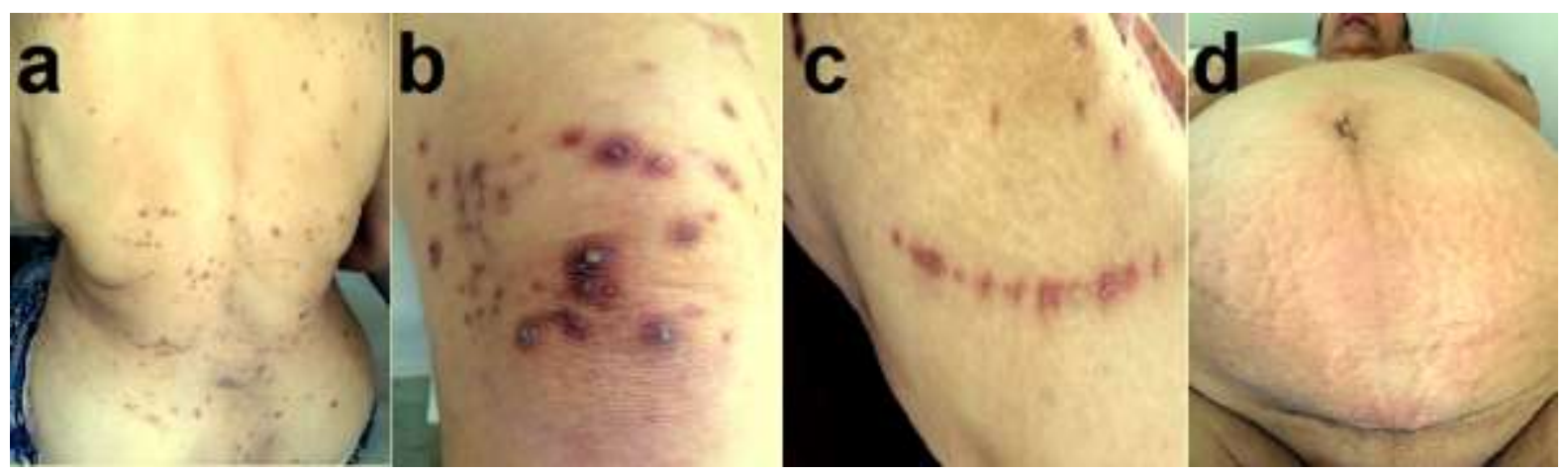

Fig 1 (a,b,c,d). Multiple scratch marks, purple and umblicated papulonodular lesions on the back and extensor surfaces of the extremities, and prominent vessels and multiple striae on abdominal wall.

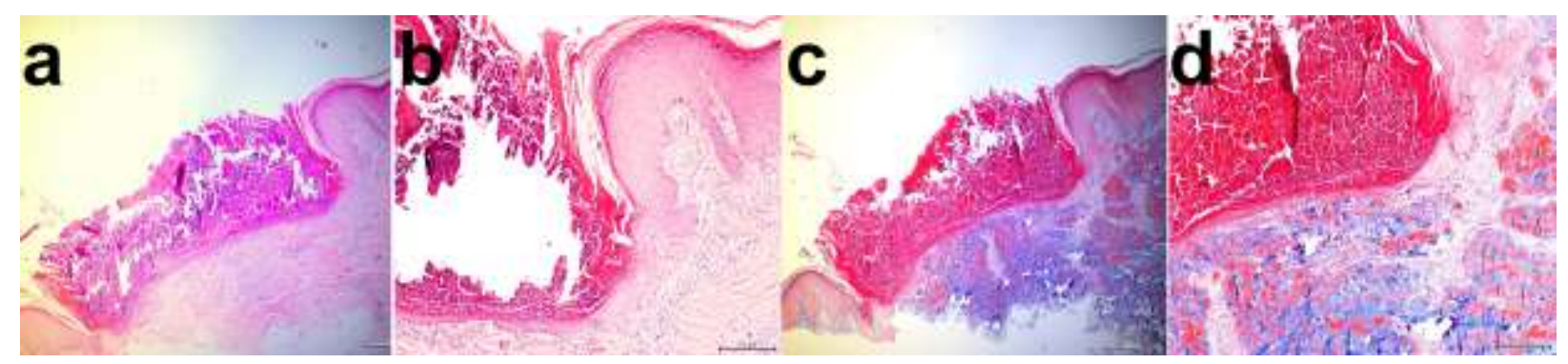

Fig 2. Invaginated necrotic epidermis which extends into the reticulated dermis, which is sharply bordered laterally by prominent epithelial hyperplasia, acanthosis, and central keratotic plug containing cellular debris and degenerated collagen (a.HEX40, b. HEX100). Coarse and degraded eosinophilic collagen bundles beneath the ulcer base and in the mid dermis (c. Masson trichromeX40, d. Masson trichromeX100) 